\title{
Preparation of a polyclonal antibody against immunogenic fragment of bovine intestinal peptide transporter I (bPepTI)
}

\author{
Xiuxin Zhao, Xueyan Lin, Guimei Liu, Zhonghua Wang* \\ College of Animal Science and Technology, Shandong Agricultural University, Tai'an, China \\ Email: ${ }^{*}$ zhwang@sdau.edu.cn
}

Received 12 January 2012; revised 28 February 2012; accepted 15 March 2012

\begin{abstract}
The goal of the current study is to prepare polyclonal antibody against bovine intestinal peptide transporter I (bPepTI) in order to develop assay for immunological assessment of protein levels. Antigenicity of the entire bPepTI was analyzed with DNAStar, and a fragment with high antigenicity (bPepTI ORF 1369 1695) was selected, cloned in pGEX-6p-1 vector, resulting in a recombinant plasmid GST-BP, which verified by double enzyme digestion and sequenced, the recombinant plasmid was introduced to BL21. Exogenous expression was induced by IPTG and validated by Western blot analysis. The recombinant protein was isolated, purified and used for production of antiserum in mice. The specificity of antiserum was evaluated with immunobloting and titer was estimated with ELISA. Results indicate that the antibody against $b$ PepTI was produced. The optimal GST-BP antigen embedding concentration was $0.5 \mu \mathrm{g} / \mathrm{ml}$. The optimal dilution was 1:400. An indirect ELISA assay indicates the effective dilution was 1:102400.
\end{abstract}

Keywords: Bovine Type I Peptide Transporter; Clone; Prokaryotes Expression; Antibody Preparation

\section{INTRODUCTION}

Evidence emerged since 1950s that animal intestine can directly absorb peptides of two or three amino acid residues ([1-6]) cloned and analyzed a proton-coupled small peptide transporter called PepTI [6]. This transporter was mainly found in gut. Further studies showed that PepTI was specific to brush border of gastrointestinal absorptive cells [7]. To date, PepTI has been cloned from rats, canines, chickens, humans, rabbits, pigs, ovine and bovine.

It has been found that certain mutations involving malfunction of amino acid transporters result in amino acid

${ }^{*}$ Corresponding author. deficiency (for example, tryptophan deficiency in Hartnup disease patient), but the symptom can be ameliorated by supplement of dipeptide [8]. Therefore, absorption of small peptide in enterocytes has physiological values, although its nutritional value is unclear. Studies on amino acid flux in gastrointestinal blood stream showed no consistent results. In calves, for example, it has been shown that small peptides constituted $79 \%$ of total amino acid absorbed into portal vein in calves [9]. In sheep, however, no small peptide was found to be absorbed into portal vein [10]. Although differences in the results among these studies are attributable to species and diet, more important reason may be due to the inaccurate nature of the flux determination methods. It was clear that the standard errors in measurements of flux differences between portal vein and arteries were huge. Differences among methods in protein preparation led to large differences in observed peptide absorption results [11-13]. The relationship between the level of transporter expression and the amount of substrate supply may, to a certain extent, clarify the importance of small peptide absorption. Studies have shown that small peptides promoted PepTI capacity [14-16] and transporter mRNA levels [17,18]. These results supported the notion that small peptide absorption had nutritional values. However, it was common for transporters to increase expression level when stimulated by their substrates. More convincing evidence may come from PepTI expression elevation seen in glucose transporter under the stimulation of substrate. Unfortunately, no such studies were available to date. Also, changes in mRNA level may not be reflected in protein levels [19]. Studies on whether the substrate stimulates the transporter expression may provide further insight into small peptide uptake.

It is of interest to study how substrate stimulates PepTI mRNA and protein express further. Establishment of an appropriate assay for PepTI protein level is an indispensible step. Protein levels are measured either directly or indirectly. Direct methods require the extraction and purification of proteins directly from tissues. Indirect me- 
thods include immunohistochemistry [20], Western blot [7], and ELISA. Indirect approaches require specific antibody. Due to the complexity of protein composition in intestinal mucosa, it is difficult to isolate PepTI. So indirect detection is more applicable. Therefore, it is necessary to prepare antibody. Currently, synthetic peptides have been used for human and mouse PepTI antibody preparation [21,22]. Here, we report the preparation of antibody against bovine PepTI using recombinant $b$ PepTI expressed in prokaryote as antigen.

\section{MATERIALS AND METHODS}

\subsection{Sample Preparation}

Healthy Limousin $\times$ Luxi hybrid calves were sacrificed in beef processing plant and rumen tissue samples were collected, washed in saline, and then placed on ice-cold glass plate. Rumen epithelial cells were peeled off and transferred to autoclaved $1.5 \mathrm{ml}$ tubes, and frozen in liquid nitrogen. The entire procedure from sacrifice to tissue frozen did not exceed $1 \mathrm{~h}$. The frozen tissues were transferred to lab and store at $-70^{\circ} \mathrm{C}$.

\subsection{RT-PCR}

Total RNA was isolated with Trizol and evaluated with electrophoresis and A260/A280 ratio. Total RNA showed three clear bands in gel. The A260/A280 ratio was between 1.8 and 2.0. These datas indicate the good quality of total RNA. The structure of $b$ PepTI was analyzed with DNA star software. A segment (designated as BP, derived from nucleotide position $1369-1695 \mathrm{bp}$ in the ORF) of high antigenicity was selected and amplified following the manual of Takara RNA PCR kit Ver 3.0. The primers were: P1 5'CGCGGATCCCATCGCCATACCCTTCTCGTC-3', and P2 5'-CCGCTCGAGGGGGCAACCGT TCACTCTTTC-3'. Restriction sites for BamHI and XhoI were separately introduced into the primers. PCR thermal cycles was consisted of pre-denature at $94^{\circ} \mathrm{C}$ for 2 min, 30 cycles of amplification $\left(94^{\circ} \mathrm{C} 30 \mathrm{~s}, 61.7^{\circ} \mathrm{C} 30 \mathrm{~s}\right.$, $72^{\circ} \mathrm{C} 30 \mathrm{~s}$ ) and a final extension at $72^{\circ} \mathrm{C}$ for $2 \mathrm{~min}$.

\subsection{Construction and Expression of Recombinant Vector}

Construction of prokaryotic expression vector GST-BP. The amplified target sequence was purified, cloned in pMD18-T vector. The vector was propagated in host cell DH5 $\alpha$. Then, the target fragment was transferred to expression vector pGEX-6p-1 after both vectors were double digested with BamHI and XhoI. Recombinant colonies were analyzed with PCR, double digestion and sequencing, which confirmed that the target sequence was correctly inserted in the vector.

Expression and characterization of the recombinant plasmid. The positive plasmid was used to transform host BL21. BL21 is one kind of E.coli bacterial cells. Positive transformant was propagated and induced for $6 \mathrm{~h}$ with IPTG at a final concentration $0.1 \mathrm{mM}$. The resulting culture was disrupted with lysis buffer, lysozyme and ultrasound, examined with SDS-PAGE. The solubility of the expression product was examined. The insoluble portion (inclusion body) was dissolved with urea for further analysis. The soluble portion was analyzed directly.

Western blot analysis of the target peptide. Membrane was blocked with $5 \%$ skim milk at $4^{\circ} \mathrm{C}$ overnight, incubated with mouse anti-GST monoclonal antibody for $2 \mathrm{~h}$, and then with HRP-conjugated goat-anti-mouse IgG for $1 \mathrm{~h}$. Finally, color was developed by incubation with DAB for 5 - $30 \mathrm{~min}$, and color development was terminated with distilled water.

\subsection{Polyclonal Antibody Preparation and Characterization}

Immunization. The target protein was isolated with SDSPAGE, negatively stained with pre-chilled KCL $(0.25 \mathrm{M})$ and the target band (white) was collected, freeze-dried, and grinded. The powder was mixed with $1 \times$ PBS of equal volume. The preparation was injected under skin of abdomen and back of mice (100 $\mu \mathrm{g} /$ mouse). Immunization was boosted every two weeks for three times. The negative control mice were injected with saline.

Antiserum collection. One week after the third boost, blood was collected from caudal vein, settled for $1 \mathrm{~h}$ at $37^{\circ} \mathrm{C}$ and transferred to $4^{\circ} \mathrm{C}$ overnight. Serum was separated by centrifugation at $4000 \mathrm{rpm}$ for $10 \mathrm{~min}$, stored at $-20^{\circ} \mathrm{C}$ until use.

Antibody specificity analysis. The constructed vector pET-32a-BP (His-BP, for short) was expressed in BL21 (DE3), which is one kind of E.coli bacterial cells. The recombinant protein was verified with mouse anti-His tag monoclonal antibody. After verification, the recombinant protein was used for antiserum verification. Specificity of the antibody was analyzed with Western blot, GST-BP fusion protein, GST vector protein, and His-BP fusion protein as antigens. The antiserum was used as the primary antibody.

Antibody titer analysis. The soluble GST-BP fusion protein was used as antigen, and diluted with embedding buffer to final concentrations of $8,2,0.5,0.1$ and 0.02 $\mu \mathrm{g} / \mathrm{mL}$. Antiserum and negative serum were diluted to 1:100, 1:200, 1: 400, 1:800, 1:1600. Color was developed with TMB, and stopped by addition of $2 \mathrm{M} \mathrm{H}_{2} \mathrm{SO}_{4}$. Absorbance was measured at $450 \mathrm{~nm}$ with ELISA reader. And the optimal antigen embedding concentration and optimal antibody dilution were determined [23].

Antigen was embedded at the optimal concentration. Antiserum and control serum were diluted to $1: 400,1$ : 
$1600,1: 6400,1: 25600,1: 102400,1: 409600$ and $1:$ 1638400. Optical density was measured at $450 \mathrm{~nm}$ with ELISA reader for sample $(\mathrm{P})$, negative control $(\mathrm{N})$, blank control (B). The titer of antiserum was defined as the ratio of $(\mathrm{P}-\mathrm{B}) /(\mathrm{N}-\mathrm{B})$ greater than 2.1 .

\section{RESULTS}

\subsection{Amplification of BP Fragment from bPepTI}

Sequencing of the amplified product showed that the insert was the target fragment (BP fragment) of $327 \mathrm{bp}$ (Figure 1). Compared with GenBank record (accession BC140526), the amplified BP fragment had three base pair changes: 99 (T-C), 252 (A-G), and 265 (C-T). The first two changes were synonymous and the last change was non-synonymous (Pro89Ser).

\subsection{Construction of Prokaryotic Expression Vector}

The BP fragment was cloned in pre-defined orientation into pGEX-6p-1 vector. The recombinant plasmids GSTBP and His-BP were double-digested with BamHI and XhoI, resulting in a fragment between 250 and 500 bp in size, which was the expected (Figures 2, 3). These results indicate that the right plasmids were obtained.

\subsection{Detection of Recombinant Protein}

\subsubsection{SDS-PAGE Analysis of Recombinant Protein}

Figure 4 showed the expressed recombinant protein in SDS-PAGE gel. Bacterial culture with the null vector pGEX-6p-1 had a $26 \mathrm{kDa}$ band, whereas the culture with the recombinant GST-BP expressed a $38.1 \mathrm{kDa}$ band. The bacterial culture with empty vector pET-32a showed a $18.7 \mathrm{kDa}$ band, whereas the one with His-BP produced a $31 \mathrm{kDa}$ band. These bands were consistent with expected sizes, indicating that the fusion proteins were

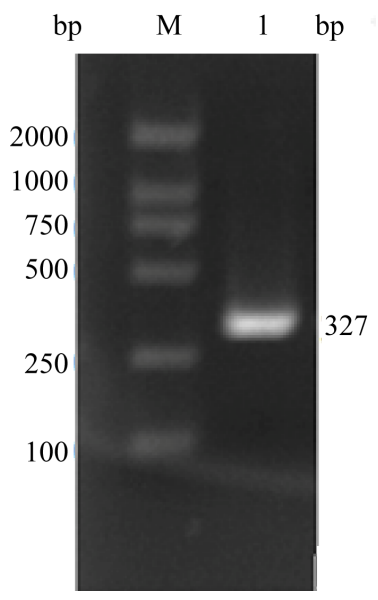

Figure 1. Target fragment.

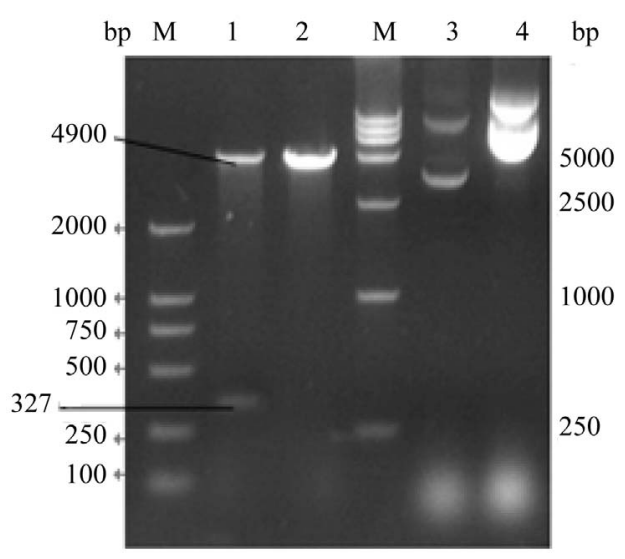

Figure 2. Product of double digested GST-BP. 1. GST-BP digested with BamHI and XhoI; 2. pGEX6p-1 digested with BamHI and XhoI; 3. GST-BP recombinant plasmid; 4. pGEX-6p-1 plasmid.

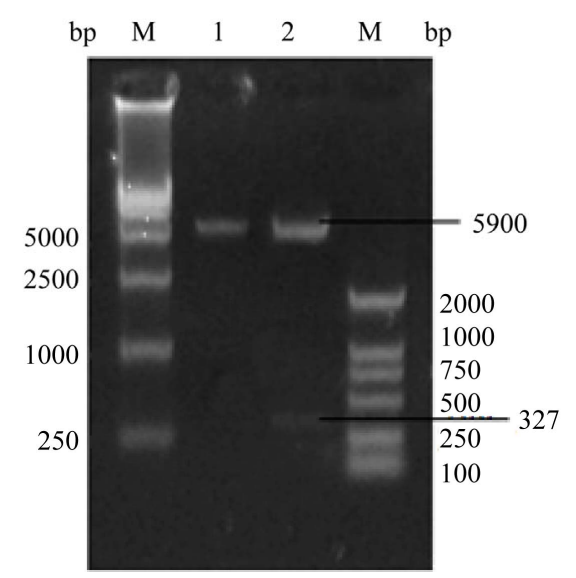

Figure 3. Product of double digested HisBP. 1. pET 32a GST-BP digested with Bam$\mathrm{HI}$ and XhoI; 2. His-BP digested with Bam$\mathrm{HI}$ and $X h o \mathrm{I}$.

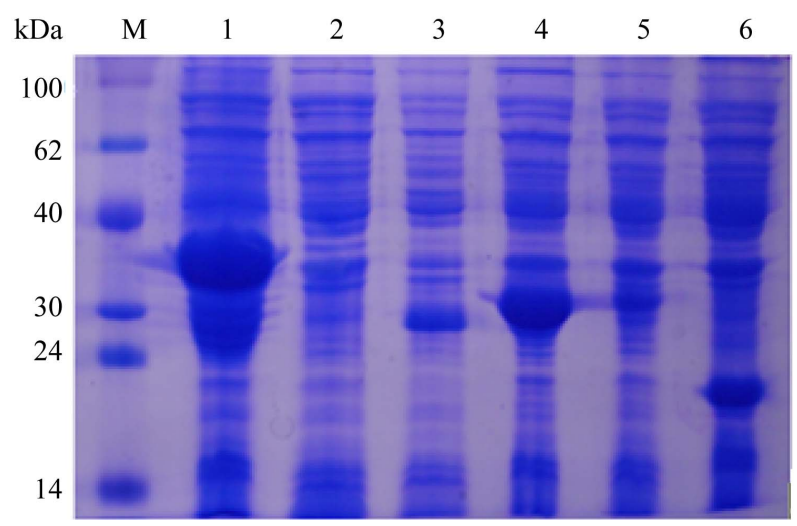

Figure 4. Recombinant protein analysis. 1. Bacteria BL21/ GST-BP induced by IPTG; 2. Bacteria BL21/GST-BP without induction; 3. Bacteria BL21/pGEX-6p-1 induced by IPTG; 4. Bacteria BL21(DE3)/His-BP induced by IPTG; 5 . Bacteria BL21 (DE3)/His-BP without induction; 6. Bacteria BL21 (DE3)/pET32a induced by IPTG. 
successfully produced. The fusion proteins were $47.3 \%$ and $29.3 \%$ of the total bacterial proteins, as indicated by analysis with BandScan 5.0 software. These levels of expression were of high efficient.

\subsubsection{Solubility of Recombinant Proteins}

Recombinant bacteria were disrupted with ultrasound, and then centrifuged. The supernatant and pellet were analyzed with electrophoresis (Figure 5). Results showed that recombinant proteins were mainly in the pellet as inclusion body.

\subsubsection{Western Blot Analysis of Recombinant Proteins}

The results of Western blot analysis were shown in Figures 6, 7. Bacterial culture with GST-PB plasmid showed a specific band of about $30-40 \mathrm{KDa}$ when detected with anti-GST antibody. Similarly, bacterial culture with HisBP showed a specific band of $30 \mathrm{KDa}$ when detected with Anti-His antibody. These results indicate that the targeted fragment was expressed.

\subsection{Antibody Specificity}

GST-BP and His-BP fusion proteins as antigen, Western blot analysis using antiserum prepared in this study showed a $38.1 \mathrm{KDa}$ and a $31 \mathrm{KDa}$ band, respectively (Figure 8), suggesting that specific polyclonal antibody against target BP fragment and GST tag was produced.

\subsection{Titer Analysis}

The titer of the antiserum was determined with ELISA using purified GST-BP protein as antigen. Positive serum had an $\mathrm{OD}_{450}$ reading of about 1 , while the negative control serum had an $\mathrm{OD}_{450}$ reading of about 0.1. Based on maximal $\mathrm{P} / \mathrm{N}$ criterion, the optimal antigen embedding

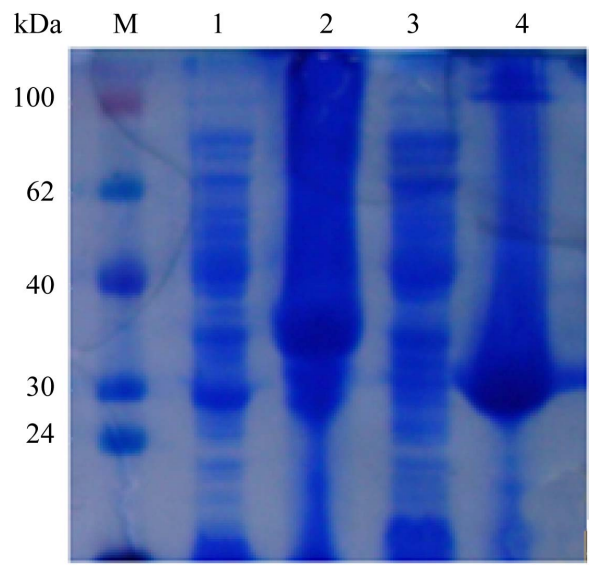

Figure 5. Recombinant bacteria disrupted by ultrasound. 1. GST-BP recombinant, supernatant; 2. GST-BP recombinant, pellet; 3. His$\mathrm{BP}$ recombinant, supernatant; 4. His-BP recombinant, pellet.

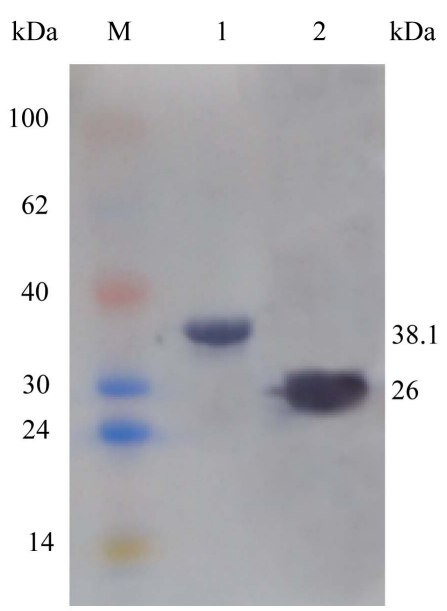

Figure 6. Protein with GST-Tag expressed in E.coli BL21. 1. GST-BP fusion protein; 2. pGEX-6p-1 vector.

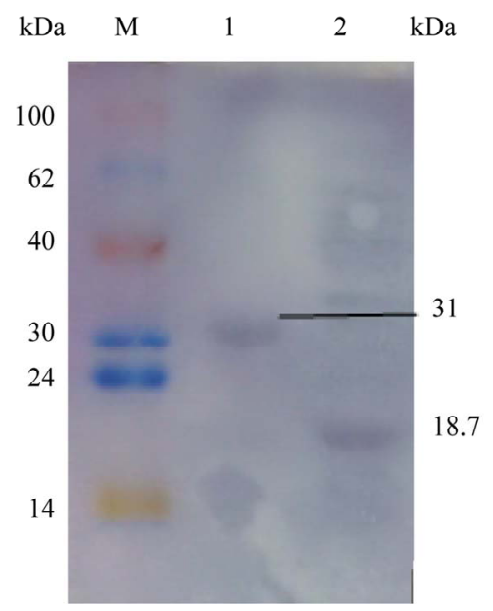

Figure 7. Protein with His-Tag expressed in E.coli BL21 (DE3). 1. HisBP fusion protein; 2. pET-32a vector.

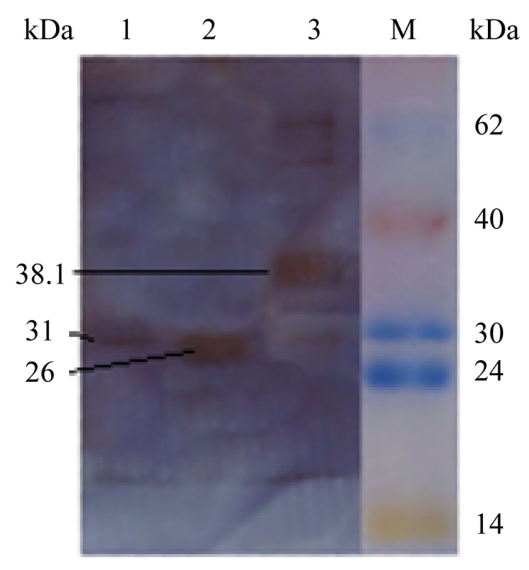

Figure 8. Specificity of polyclonal antibody. 1. His-BP Fusion protein; 2. pGEX$6 \mathrm{p}-1$ empty vector; 3 . GST-BP fusion protein; 4. Pre-stained marker. 
concentration was $0.5 \mu \mathrm{g} / \mathrm{ml}$, and the optimal dilution of the antiserum was 1:400. Indirect ELISA test results indicated that the antibody was effective at 1:102400 dilutions.

\section{DISCUSSION}

PepTI is a transmembrane transporter. Results from hydrophobicity analysis (Kyte-Doolottle) indicate that $b$ PepTI has 12 transmembrane motifs, which is identical to that found in rabbit [6], ovine [24], and bird [25]. We attempted to express the entire protein in prokaryotes, but it was unsuccessful. This difficulty may be due to the transmembrane and hydrophobic region of the protein. We later analyzed the antgenicity of the protein and selected a fragment of high antigenicity for expression in prokaryote [26].

Antigenic epitopes in $b$ PepTI are concentrated between amino acid residues 400 - 600. The current trial selected the peptide containing residues 378 - 548. This fragment is the largest extracellular domain in the entire protein, located between transmembrane domains 9 and 10. This fragment was expressed successfully. Other smaller extracellular domains of $b$ PepTI may also be used for antibody preparation. Antibody against these smaller domains may have better specificity. However, their antigenicity may be inferior. They may be used for diagnostic or hybridization. The goal of the current study was to prepare antibody to be used for expression analysis. Therefore, we selected the domain of high antigenicity. This domain in $b$ PepTI shares $58.7 \%, 61.5 \%, 66.1 \%$ and $92.7 \%$ amino acid sequences with those in mice, rabbit, human and goat PePTI. Since the entire $b$ PepTI shares $80.3 \%, 78.9 \%, 82.8 \%$ and $95.1 \%$ amino acid sequences, respectively, with those species mentioned above, the domain selected for antibody preparation is more divergent among species. This feature is beneficial for preparation of more specific antibodies.

Using right plasmid vector and the host cell is the key for high level expression of cloned proteins. In the current trial, the vector was Pgex-6P-1. This vector contains a Tac promoter, which is a highly effective hybrid made from $L a c$ and $\operatorname{Trp}$ [27]. BL21 is an expression system suitable for non-T7 promoters (Tac promoter, such as pGEX, PMal). TL21(DE3) is suitable for T7 (pET), T7/ Lac expression. In order to increase the express effect, BL21 was used to express GST-BP and BL21 (DE3) was used to express His-BP recombinant protein.

Small peptides (basically less than 15 amino acid residues) was routinely used for producing Polyclonal antibodies, and purified natural proteins, and recombinant large peptides as the antigen. Small peptides are immunoreactive, but weak or even lack of antigenicity. Therefore, small peptides are normally conjugated with large carrier protein to produce complete antigen [28]. Be- cause the immune properties are determined by spatial structure of protein, synthetic peptide has a high failure rate for antibody production compared with large peptide expression in vitro. To produce antibody in use of natural protein, purification of easily accessible natural protein sources is required. This approach is not feasible to $b$ PepTI. Therefore, in the current trial, the recombinant large peptide approach was used. The prokaryotic expression system allowed the production of large amount within a short period of time, which is beneficial for the purification and analysis.

Animals that used for producing antibody in laboratory are usually rabbits or mice. It is generally thought that the greater the difference between the antigen and host, the better the immunogenicity. The gastroinstestinal tract of rabbit is more similar to bovine's. $b$ PepTI shares $61.5 \%$ and $58.7 \%$ amino acid sequence with rabbit and mice respectively. Therefore, mice were selected for immunization to obtained greater immunogenicity.

The antigen used for immunization had a GST tag. In order to exclude the possibility that the antiserum is against GST only, His-BP fusion protein that has the same epitope was expressed. His-BP fusion protein was reactive with the antiserum, indicating the antiserum had antibody against $b$ PepTI.

\section{CONCLUSION}

We have got the polyclonal antibody against a fragment $b$ PepTI ORF 1369 - 1695 of bovine intestinal peptide transporter I (bPepTI).

\section{ACKNOWLEDGEMENTS}

This work is supported by the earmarked fund for Modern Agro-industry Technology Research System of China and China natural science fund.

\section{REFERENCES}

[1] Newey, H. and Smyth, D.H. (1959) The intestinal absorption of some dipeptides. Journal of Physiology, 145, 48-56.

[2] Newey, H. and Smyth, D.H. (1960) Intracellular hydrolysis of dipeptides during intestinal absorption. Journal of Physiology, 152, 367-380.

[3] Adibi, S.A. and Morse, E.L. (1971) Intestinal transport of dipeptides in man: Relative importance of hydrolysis and intact absorption. Journal of Clinical Investigation, 50, 2266-2275. doi:10.1172/JCI106724

[4] Boullin, D.J., Crampton, R.F., Christine, E.H. and Pelling, D. (1973) Intestinal absorption of peptides containing glycine, phenylalanine, proline, $\beta$-alanine or histidine in the rat. Clinical Science and Molecular Medicine, 45, 849-858.

[5] Matthews, J.C. and Webb, K.E. Jr. (1995) Absorption of 
L-carnosine, L-methionine, and L-methionylglycine by isolated sheep ruminal and omasal epithelial tissue. Journal of Animal Science, 73, 3463-3475.

[6] Fei, Y.J., Kanai, Y., Nussberger, S., Ganapathy, V., Leibach, F.H., Romero, M.F., Singh, S.K., Boron, W.F. and Hediger, M.A. (1994) Expression cloning of a mammalian proton-coupled oligopeptide transporter. Nature, $\mathbf{3 6 8}$, 563-566. doi:10.1038/368563a0

[7] Ogihara, H., Saito, H., Shin, B.C., Terado, T., Takenoshita, S., Nagamachi, Y., Inui, K. and Takata, K. (1996) Immuno-localization of $\mathrm{H}+$ /peptide cotransporter in rat digestive tract. Biochemical and Biophysical Research Communications, 220, 848-852. doi:10.1006/bbrc.1996.0493

[8] Asatoor, A.M., Cheng, B., Edwards, K.D.G., Lant, A.F., Matthews, D.M., Miline, M.D., Navab, F. and Richard, A.J. (1970) Intestinal absorption of dipeptides in Hartnup disease. Gut, 11, 380-387. doi:10.1136/gut.11.5.380

[9] Koeln, L.L., Schlagheck, T.G. and Webb, K.E. (1993) Amino acid flux across the gastrointestinal tract and liver of calves. Journal of Dairy Science, 76, 2275-2285. doi:10.3168/jds.S0022-0302(93)77564-5

[10] Backwell, F.R., Hipolito-Reis, M., Wilson, D. and Bruce, L.A. (1997) Quantification ofirculating peptides and assessment of peptide uptake across the gastrointestinal tract of sheep. Journal of Animal Science, 75, 3315-3322.

[11] Galibois, I., Pitre, F., Parent, G. and Savoie, L. (1991) Analysis of bound amino acids in the plasma of fed rats: A new preparation procedure. Journal of Nutrition Biochemistry, 2, 25-30. doi:10.1016/0955-2863(91)90045-7

[12] Webb, K.E. Jr., Matthews, J.C. and Dirienzo, D.B. (1992) Peptide absorption: A review of current concepts and future perspectives. Journal of Animal Science, 70, 32483257.

[13] Seal, C.J. and Parker, D.S. (1996) Effect of intraruminal propionic acid infusion on metabolism of mesenteric- and portal-drained viscera in growing steers fed a forage diet: I Ammonia, urea, amino acid, and peptides. Journal of Animal Science, 74, 245-256.

[14] Ferraris, R.P., Diamond, J. and Kwan, W.W. (1988) Dietary regulation of intestinal transport of the dipeptide carnosine. American Journal of Physiology, 255, G143G150.

[15] Brandsch, M., Ganapathy, V. and Leibach, F.H. (1995) $\mathrm{H}(+)$-peptide cotransport in Madin-Darby canine kidney cells: Expression and calmodulin dependent regulation. American Journal of Physiology, 268, F391-F397.

[16] Adibi, S.A. (1997) The oligopeptide transporter (Pept-1) in human intestine: Biology and function. Gastroenterology, 113, 332-340. doi:10.1016/S0016-5085(97)70112-4

[17] Ogihara, H., Suzuki, T., Nagamachi, Y., Inui, K. and Takata, K. (1999) Peptide transporter in the rat small intes- tine: Ultrastructural localization and the effect of starvation and administration of amino acids. Histochemical Journal, 31, 169-174. doi:10.1023/A:1003515413550

[18] Shiraga, T., Miyamoto, K., Tanaka, H., Yamamoto, H., Taketani, Y., Morita, K., Tamai, I., Tsuji, A. and Takeda, E. (1999) Cellular and molecular mechanisms of dietary regulation on rat intestinal $\mathrm{H}+/$ Peptide transporter PepT1. Gastroenterology, 116, 354-362. doi:10.1016/S0016-5085(99)70132-0

[19] Shen, H., Smith, D.E. and Brosius, F.C. III. (2001) Developmental expression of PepT1 and PepT2 in rat small intestine, colon, and kidney. Pediatric Research, 49, 789-795. doi:10.1203/00006450-200106000-00013

[20] Hussain, I., Kellett, G.L., Affleck, J., Shepherd, E.J. and Boyd, C.A.R. (2001) Expression and cellular distribution during development of the peptide transporter (PepT1) in the small intestinal epithelium of the rat. Cell and Tissue Research, 307, 139-142.

[21] Saito, H., Okud, M., Terada, T., Sasaki, S. and Inui, K. (1995) Cloning and characterization of a rat $\mathrm{H}+/$ peptide cotransporter mediating absorption of beta-lactam antibiotics in the intestine and kidney. Journal of Pharmacology and Experimental Therapeutics, 275, 1631-1637.

[22] Ziegler, T.R., Esyivariz, C.F., Gu, L.H., Bazargan, N., Umeakunne, K., Wallace, T.M., Diaz, E.E., Rosado, K.E., Pascal, R.R., Galloway, J.R., Wilcox, J.N. and Leader, L.M. (2002) Distribution of the $\mathrm{H}+$ /peptide transporter PepT1 in human intestine: Up-regulated expression in the colonic mucosa of patients with short-bowel syndrome1. American Journal of Clinical Nutrition, 75, 922-930.

[23] Coligan, J.E. (2009) A laboratory manual for immunology. Science Press, Beijing, 23-30.

[24] Pan, Y.X., Eric, A.W., Jeffrey, R.B. and Webb, K.E. Jr. (2001) Expression of a cloned ovine gastrointestinal peptide transporter (oPepT1) in xenopus oocytes induces uptake of oligopeptides in vitro. Nature, 131, 1264-1270.

[25] Chen, H., Pan, Y.X., Wong, E.A., Bloomquist, J.R. and Webb, K.E. Jr. (2002) Molecular cloning and functional expression of a chicken intestinal peptide transporter (cPepT1) in xenopus oocytes and Chinese hamster ovary cells. Nature, 132, 387-393.

[26] Zhou, Y., Wu, F., Fan, G.C., Wu, Z.Z. and Wang, Q.M. (2009) New methods of protein antibody preparation on account of the epitope of antigen. Current Immunology, 29, 364-369.

[27] Chen, J.Z. and Xue, J.L. (2007) Vectors and genetic manipulation. 54-64.

[28] Kapsenberg, M.L. (1996) Chemicals and proteins as allergens and adjuvants. Toxicology Letters, 86, 79-83. doi:10.1016/0378-4274(96)03676-4 